\title{
Pointwise limit theorem for a class of unbounded operators in $\mathbb{L}^{r}$-spaces
}

\author{
by \\ RYSZARD JAJTE (Łódź)
}

\begin{abstract}
We distinguish a class of unbounded operators in $\mathbb{L}^{r}, r \geq 1$, related to the self-adjoint operators in $\mathbb{L}^{2}$. For these operators we prove a kind of individual ergodic theorem, replacing the classical Cesàro averages by Borel summability. The result is equivalent to a version of Gaposhkin's criterion for the a.e. convergence of operators. In the proof, the theory of martingales and interpolation in $\mathbb{L}^{r}$-spaces are applied.
\end{abstract}

1. Introduction. The paper is an attempt of extension of the pointwise ergodic theory in $\mathbb{L}^{r}(\mu)$-spaces [10] to the case of some unbounded operators. Some questions are maybe touched here for the first time and perhaps new techniques will have to be developed. We start by trying to follow some ideas and methods known in the case of $\mathbb{L}^{2}(\mu)$. That is why we confine ourselves to a class of linear maps in $\mathbb{L}^{r}$ related to unbounded self-adjoint operators in $\mathbb{L}^{2}$. To discuss the asymptotic properties of unbounded operators we pass from Cesàro averages to Borel summability. Let us recall the definition.

We say that a sequence $x=\left\{\xi_{k}\right\}$ of numbers (or vectors in a Banach soace) is summable to $\xi$ by the Borel method if

$$
\lim _{m \rightarrow \infty} e^{-m} \sum_{k=0}^{\infty} \frac{m^{k}}{k !} \xi_{k}=\xi .
$$

Then we write $B$-lim $\xi_{k}=\xi$. The sequence

$$
B(m, x)=e^{-m} \sum_{k=0}^{\infty} \frac{m^{k}}{k !} \xi_{k}, \quad m=1,2, \ldots,
$$

is called the Borel transform of $x$.

2000 Mathematics Subject Classification: Primary 47A35, 60F15; Secondary 40G10, 47B40.

Key words and phrases: individual ergodic theorem, martingale transform, square function, Burkholder interpolation inequality in $\mathbb{L}^{r}$-spaces, Borel summability. 
In what follows, for $\zeta=\left\{\lambda^{k}\right\}$, we shall often write, for convenience, $B(m, \lambda)$ instead of $B(m, \zeta)$.

Let $\left(\mathcal{F}_{t}, t \in \mathbb{R}\right)$ be a filtration in a probability space $(\Omega, \mathcal{F}, \mu)$. For $r \geq 1$, in $\mathbb{L}^{r}(\Omega, \mathcal{F}, \mu)$ one can consider (unbounded) operators of the form

$$
A=\int_{\mathbb{R}} t d E_{t}
$$

where $E_{t}=\mathbb{E}\left(\cdot \mid \mathcal{F}_{t}\right)$ is the conditional expectation operator.

The class of such operators is a natural and important counterpart of self-adjoint operators in $\mathbb{L}^{2}(\mu)$.

For bounded operators in $\mathbb{L}^{r}(1<r<\infty)$ of the form $(0)$, several individual ergodic theorems can be proved [12]. On the other hand, in the case of $\mathbb{L}^{2}(\mu)$ some pointwise limit theorems can be proved for unbounded (normal) operators [11], [13].

Our aim is to prove a theorem concerning operators of the form (0) in $\mathbb{L}^{r}(\mu), 1<r<\infty$.

Let us begin with some notation. We fix a probability space $(\Omega, \mathcal{F}, \mu)$ and a filtration $\left(\mathcal{F}_{t}\right),-\infty<t \leq 1$, in $(\Omega, \mathcal{F})$ satisfying the conditions:

(i) $\bigcap_{s>t} \mathcal{F}_{s}=\mathcal{F}_{t}$ for $-\infty<t<1$,

(ii) $\sigma\left(\bigcup_{t<1} \mathcal{F}_{t}\right)=\mathcal{F}_{1}=\mathcal{F}$,

(iii) $\mathcal{F}_{-\infty}=\{\emptyset, \mathcal{F}\}$.

Let us write formally

$$
A=\int_{-\infty}^{1} t d E_{t},
$$

where $E_{t}$ is the conditional expectation

$$
E_{t}=E\left(\cdot \mid \mathcal{F}_{t}\right), \quad-\infty<t \leq 1 .
$$

In $\widehat{\mathbb{L}}^{2}=\mathbb{L}^{2} \ominus$ [const], $\left(E_{t}\right)$ is a resolution of the identity, and (1) defines a self-adjoint operator in $\widehat{\mathbb{L}}^{2}$.

Since the operators $E_{t}$ are defined in $\mathbb{L}^{r}$-spaces, $r \geq 1$, the operator $A$ can also be considered in these spaces as an unbounded operator.

We set

$$
\widehat{\mathbb{L}}^{r}=\left\{\xi \in \mathbb{L}^{r}: \int_{\Omega} \xi d \mu=0\right\} \quad \text { for } r \geq 1 .
$$

The family of operators (2) is a uniformly bounded (spectral) family of projections on $\widehat{\mathbb{L}}^{r}$, satisfying the conditions

(a) $E_{s} E_{t}=E_{t} E_{s}=E_{\min (s, t)},-\infty<s, t \leq 1$,

(b) $E_{-\infty}=0, E_{1}=I$,

(c) $E_{t}$ is right continuous in the strong operator topology for $t \in(-\infty, 1)$ and has a strong left limit $E(t-)$ at each point $-\infty<t \leq 1$. 
In the following we shall write $E\{a<t<b\}=E_{b-}-E_{a}$.

The operator (1) can be defined on $\widehat{\mathbb{L}}^{r}$ as follows. We first define a bounded operator $\int_{a}^{b} f(t) d E_{t}$, for $-\infty<a<b<\infty$, where $\left(E_{t}\right)$ is the spectral family determined by the filtration $\left(\mathcal{F}_{t}\right)$, i.e. $E_{t}=E\left(\cdot \mid \mathcal{F}_{t}\right)$. We assume that $f$ is a function of bounded variation on $[a, b]$.

Given a partition

$$
\pi=\left(a=t_{0}<t_{1}<\ldots<t_{n}=b\right)
$$

of the interval $[a, b]$, we put

$$
\pi(f)=\sum_{i=1}^{n} f\left(t_{i}\right)\left(E_{t_{i}}-E_{t_{i-1}}\right) .
$$

The Riemann-Stieltjes sums $\pi(f)$ converge in the strong operator topology as $\pi$ runs through the set of partitions of $[a, b]$ directed by refinement (cf. [6], [3]). This strong limit of $\pi(f)$ is denoted by $\int_{a}^{b} f(t) d E_{t}$.

Having defined $\int_{a}^{b} t d E_{t}$ as a bounded linear operator on $\widehat{\mathbb{L}}^{r}$, for the operator $A$ in (1) we set

$$
D(A)=\left\{\xi \in \widehat{\mathbb{L}}^{r}: s-\lim _{d \rightarrow-\infty} \int_{d}^{1} t d E_{t} \xi \text { exists }\right\}
$$

and

$$
A \xi=\lim _{d \rightarrow-\infty} \int_{d}^{1} t d E_{t} \xi \quad \text { for } \xi \in D(A) .
$$

We shall need one more definition. Let $A$ be an unbounded operator acting in a Banach space $X$. A vector $x \in X$ is said to be analytic for $A$ if $x \in$ $\bigcap_{n \geq 1} D\left(A^{n}\right)$ and the function

$$
z \mapsto \sum_{n \geq 0} \frac{\left\|A^{n} x\right\|}{n !} z^{n}
$$

is entire (cf. [4], [16]).

2. Main result. Now we are in a position to formulate our result.

TheOREm. Let $(\Omega, \mathcal{F}, \mu)$ be a probability space and let $\left(\mathcal{F}_{t},-\infty<t \leq 1\right)$ be a filtration in $(\Omega, \mathcal{F})$. Fix an arbitrary $1<r<\infty$. Let

$$
A=\int_{-\infty}^{1} t d E_{t}
$$

be the "spectral representation" of an operator in $\widehat{\mathbb{L}}^{r}(\Omega, \mathcal{F}, \mu)$, where $E_{t}=$ $E\left(\cdot \mid \mathcal{F}_{t}\right)$ is the conditional expectation. If a vector $\xi \in \widehat{\mathbb{L}}^{r}(\mu)$ is analytic 
for A, then

$$
B-\lim _{n \rightarrow \infty} A^{n} \xi=E\{1\} \xi \quad \mu \text {-a.e. }
$$

Let us note that for $r=2$ the theorem is an immediate consequence of [11, Corollary 3.2], by the martingale convergence theorem.

It is a matter of taste to consider the operator $A$ in $\mathbb{L}^{r}$ or in $\widehat{\mathbb{L}}^{r}$. Passing from $\mathbb{L}^{r}$ to $\widehat{\mathbb{L}}^{r}$ and conversely is always trivial. In $\widehat{\mathbb{L}}^{r}$ any filtration leads to the "spectral" family of projections and then the connections with normal operators in $\mathbb{L}^{2}$ are clearer.

3. Proof of the theorem. The proof is rather long but its general idea can be described as follows. Roughly speaking, we replace orthogonality arguments employed in $\mathbb{L}^{2}(\mu)$ for, say, self-adjoint operators by estimates of square functions of suitable martingale transforms. To improve these estimates to be strong enough for our purpose, we use interpolation techniques (cf. [2]).

The proof will be reduced to a version of Gaposhkin's criterion for almost sure convergence [8], [9], [2], which in our context says that (3) is equivalent to

$$
E\left\{1-2^{-n}<t<1\right\} \xi \rightarrow 0 \quad \mu \text {-a.e. as } n \rightarrow \infty .
$$

Having the above equivalence we readily get (3) because (4) follows from the martingale convergence theorem.

Clearly, to prove the equivalence of (3) and (4) it suffices to show that, for $\zeta=\left(\lambda^{n}\right)$ and $x=\left(A^{n} \xi\right)$,

$$
\begin{aligned}
\alpha_{n} & =\int_{\left\{1-2^{-n} \leq \lambda<1\right\}}\left(B\left(2^{n}, \zeta\right)-1\right) d E_{\lambda} \xi \rightarrow 0 \quad \text { a.e., } \\
\beta_{n} & =\int_{\left\{-\infty<\lambda<1-2^{-n}\right\}} B\left(2^{n}, \zeta\right) d E_{\lambda} \xi \rightarrow 0 \quad \text { a.e., } \\
\gamma_{n} & =\max _{1 \leq k<2^{n}}\left|B\left(2^{n}+k, x\right)-B\left(2^{n}, x\right)\right| \rightarrow 0 \quad \text { a.e. }
\end{aligned}
$$

It should be stressed here that $\xi$ being an analytic vector for $A$ guarantees the existence of all Borel transforms appearing above and in the following. In particular, a fairly standard argument leads to the formula

$$
B(m, x)=\int_{-\infty}^{1} B(m, \zeta) d E_{\lambda} \xi,
$$

that is,

$$
\sum_{k \geq 0} \frac{m^{k}}{k !} A^{k} \xi=\int_{-\infty}^{1} \sum_{k \geq 0} \frac{m^{k}}{k !} \lambda^{k} d E_{\lambda} \xi .
$$

This will be frequently used without any reference. 
We set

$$
I(f)=\int_{0}^{1} f(\lambda) d E_{\lambda} \xi
$$

Then, in particular,

$$
\alpha_{n}=I\left(f_{n}\right), \quad \text { where } \quad f_{n}(\lambda)=\left(B\left(2^{n}, \lambda\right)-1\right) \chi_{\left[1-2^{-n}, 1\right)}(\lambda) .
$$

Take a sequence

$$
\pi_{N}=\left(0=t_{1}^{(N)}<\cdots<t_{k(N)}^{(N)}=1\right), \quad N=1,2, \ldots,
$$

of partitions of $[0,1]$ such that, putting

$$
\pi_{N}(f)=\sum_{i=1}^{k(N)} f\left(t_{i}^{(N)}\right)\left(E_{t_{i}^{(N)}}-E_{t_{i-1}^{(N)}}\right) \xi
$$

we have, for some $D$,

$$
\left\|\left\{\sum_{n=1}^{N}\left(\pi_{N}\left(f_{n}\right)-\alpha_{n}\right)^{2}\right\}^{1 / 2}\right\|_{r} \leq D<\infty \quad \text { for } N=1,2, \ldots
$$

Then, for $1<r<\infty$,

$$
\begin{aligned}
\left\|\left\{\sum_{n=1}^{N} \alpha_{n}^{2}\right\}^{1 / 2}\right\| & \leq\left\|\left\{\sum_{n=1}^{N}\left(\alpha_{n}-\pi_{N}\left(f_{n}\right)\right)^{2}\right\}^{1 / 2}\right\|_{r}+\left\|\left\{\sum_{n=1}^{N}\left(\pi_{N}\left(f_{n}\right)\right)^{2}\right\}^{1 / 2}\right\|_{r} \\
& \leq D+\left\|\left\{\sum_{n=1}^{N}\left(\pi_{N}\left(f_{n}\right)\right)^{2}\right\}^{1 / 2}\right\|_{r}, \quad n=1,2, \ldots
\end{aligned}
$$

Thus, to prove (5) it is enough to show that

$$
\Delta_{N}=\left\|\left\{\sum_{n=1}^{N}\left(\pi_{N}\left(f_{n}\right)\right)^{2}\right\}^{1 / 2}\right\|_{r} \leq C_{r}\|\xi\|_{r} \quad \text { for } N=1,2, \ldots
$$

(here and below, $C_{\beta}$ denotes a constant depending only on the parameter $\beta$, which may be different in different places).

To this end we write

$$
\left[1-2^{-n}, 1\right)=\bigcup_{k=0}^{\infty}\left[1-2^{-n-k}, 1-2^{-n-k-1}\right)=\bigcup_{k=0}^{\infty} I_{n, k} .
$$

Then

$$
\Delta_{N} \leq \sum_{k=0}^{\infty}\left\|\left\{\sum_{n=1}^{N}\left(\sum_{t_{i}^{(N)} \in I_{n, k}} f_{n}\left(t_{i}^{(N)}\right)\right)^{2}\right\}^{1 / 2}\right\|_{r}
$$


For fixed $k$, set

$$
A_{\nu}^{(k)}=\sum_{i=1}^{\nu} \sum_{n=1}^{N} \sum_{t_{i}^{(N)} \in I_{n, k}} f_{n}\left(t_{i}^{(N)}\right)\left(E_{t_{i}^{(N)}}-E_{t_{i-1}^{(N)}}\right) \xi .
$$

Then $\left(A_{\nu}^{(k)}, \nu=1, \ldots, k(N)\right)$ is a martingale transform and, by the Burkholder inequality [5],

$$
\sup _{\nu}\left\|A_{\nu}^{(k)}\right\|_{r} \leq C_{r}\|\xi\|_{r}
$$

For fixed $k$, we let $m_{n}^{(k)}$ be such that

$$
t_{s}^{(N)} \in I_{n, k} \quad \text { if and only if } \quad m_{n}^{(k)}<s \leq m_{n+1}^{(k)} .
$$

Then $B_{n}^{(k)}=A_{m_{n}(k)}^{(k)}(n=1,2, \ldots)$ is a martingale and its square function equals

$$
Q_{k}=\left\{\sum_{n=1}^{N}\left(\sum_{T_{i}^{(N)} \in I_{n, k}} f_{n}\left(t_{i}^{(N)}\right)\left(E_{t_{i}^{(N)}}-E_{t_{i-1}^{(N)}}\right) \xi\right)^{2}\right\}^{1 / 2} .
$$

By the Burkholder inequality,

$$
\left\|Q_{k}\right\|_{r} \leq C_{r}\|\xi\|_{r}, \quad 1<r<\infty .
$$

To obtain (11), we have to improve the estimate (14), to get a good enough order of decrease of $\left\|Q_{k}\right\|_{r}$ as $k \rightarrow \infty$. To this end we interpolate some operators related to $Q_{k}$.

Notice that, for $r=2$, using the estimate $|1-B(m, \lambda)| \leq C m(1-\lambda)$ for $0 \leq \lambda \leq 1$, we readily obtain

$$
\left\|Q_{k}\right\|_{2} \leq C_{2} 2^{-k}\|\xi\|_{2}
$$

Fix $1<p<\infty$ such that $r$ lies between $p$ and 2, and define (for fixed $k$ ) an operator

$$
W_{k}^{(p)}: \mathbb{L}^{p}(\Omega, \mu) \rightarrow \mathbb{L}^{p}\left((\Omega, \mu), \ell_{N}^{2}\right)
$$

by putting

$$
W_{k}^{(p)}: \xi \mapsto\left\{\sum_{t_{s}^{(N)} \in I_{n, k}} f_{n}\left(t_{i}^{(N)}\right)\left(E_{t_{i}^{(N)}}-E_{t_{i-1}^{(N)}}\right) \xi\right\}_{n=1}^{N}
$$

Then

$$
\left\|W_{k}^{(p)}\right\|_{p} \leq C_{p}
$$

By (13), (14), (15), (17) and interpolation theorems ([1, Chapter 5]), we obtain, for some $0<\theta_{r} \leq 1$, the estimate

$$
\left\|Q_{k}\right\|_{r} \leq C_{r} 2^{-\theta_{r} k}\|\xi\|_{r}, \quad 1<r<\infty .
$$

Summing over $k$, we get (11), which together with (10) implies (5). 
To prove (6) we estimate the integral

$$
I\left(g_{n}\right)=\int_{0}^{1} g_{n}(\lambda) d E_{\lambda} \xi
$$

where $g_{n}(\lambda)=B\left(2^{n}, \lambda\right) \chi_{\left[0,1-2^{-n}\right)}(\lambda)$. We do it in a way similar to that in the proof of (5). We first approximate $I\left(g_{n}\right)$ by suitable Riemann-Stieltjes sums, say, like those in (9) and such that

$$
\left\|\left\{\sum_{n=1}^{N}\left(\pi_{N}\left(g_{n}\right)-I\left(g_{n}\right)\right)^{2}\right\}^{1 / 2}\right\|_{r} \leq D<\infty \quad \text { for } N=1,2, \ldots
$$

For $\lambda<0,|B(m, \lambda)| \leq e^{-m}$, so

$$
\sum_{n=1}^{\infty}\left\|\int_{-\infty}^{0} B\left(2^{n}, \lambda\right) d E_{\lambda} \xi\right\|_{r}^{r} \leq 2^{r}\|\xi\|_{r}^{r} \sum_{n=1}^{\infty} e^{-2^{n} r},
$$

which implies

$$
\int_{-\infty}^{0} B\left(2^{n}, \lambda\right) d E_{\lambda} \xi \rightarrow 0 \quad \text { a.e. }
$$

Thus, to prove (6) it is enough to show that, for $1<r<\infty$,

$$
\delta_{N}=\left\|\left\{\sum_{n=1}^{N}\left(\pi_{n}\left(g_{n}\right)\right)^{2}\right\}^{1 / 2}\right\|_{r} \leq C_{r}\|\xi\|_{r}, \quad N=1,2, \ldots
$$

Write

$$
\left[0,1-2^{-n}\right)=\bigcup_{k=1}^{n}\left[1-2^{-n+k}, 1-2^{-n+k-1}\right)=\bigcup_{k=1}^{N} J_{n, k} .
$$

Then

$$
\delta_{N} \leq \sum_{k=1}^{n}\left\|\left\{\sum_{n=1}^{N}\left(\sum_{t_{i}^{(N)} \in J_{n, k}} g_{n}\left(t_{i}^{(N)}\right)\left(E_{t_{i}^{(N)}}-E_{t_{i-1}^{(N)}}\right) \xi\right)^{2}\right\}^{1 / 2}\right\|_{r} .
$$

Now to get (19) we can imitate the previous argument in the proof of (11) for $\Delta_{N}$, using the estimate

$$
|B(n, \lambda)| \leq C n^{-1}(1-\lambda)^{-1} \quad \text { for } 0 \leq \lambda \leq 1 .
$$

We omit the details.

Before passing to the proof of (7) let us fix some notation. For $\zeta=\left(\lambda^{k}\right)$ and $x=\left(A^{k} \xi\right)$, we shall write briefly

$$
C_{n}=B(n, \zeta), \quad D_{n}=B(n, x), \quad \sigma_{n, m}=D_{2^{n}+m}-D_{2^{n}} .
$$


Writing $m$ in the form

$$
m=\sum_{k=1}^{n} \epsilon_{k} 2^{n-k},
$$

with $\epsilon_{k}$ equal to 0 or 1 , by the standard dyadic expansion we get

$$
\sigma_{n, m}=\sum_{k=1}^{n} \epsilon_{k} H_{n, k}^{\left(j_{k}\right)}
$$

with suitable $j_{k}$ 's, where

$$
H_{n, k}^{(j)}=\int_{(-\infty, 1)}\left[C_{2^{n}+j 2^{n-k}}(\lambda)-C_{2^{n}+(j-1) 2^{n-k}}(\lambda)\right] d E_{\lambda} \xi
$$

for $k=1, \ldots, n$ and $j=1, \ldots, 2^{k}$. Putting

$$
R_{n, k, j}=C_{2^{n}+j 2^{n-k}}-C_{2^{n}+(j-1) 2^{n-k}},
$$

we can write

$$
H_{n, k}^{(j)}=\int_{(-\infty, 1)} R_{n, k, j}(\lambda) d E_{\lambda} \xi
$$

To prove (7) we shall show that

$$
\left\|\left\{\sum_{n=1}^{N} \gamma_{n}^{2}\right\}^{1 / 2}\right\|_{r} \leq \text { const }<\infty \quad \text { for } N=1,2, \ldots
$$

We can write

$$
\left|\gamma_{n}\right| \leq \max _{\left(j_{1}, \ldots, j_{n}\right)} \sum_{k=1}^{n}\left|H_{n, k}^{\left(j_{k}\right)}\right|,
$$

where the maximum is taken over all vectors $\left(j_{1}, \ldots, j_{n}\right)$ with different entries in the set $\left\{1, \ldots, 2^{k}\right\}$. Consequently,

$$
\begin{aligned}
\gamma_{n}^{2} & \leq \max _{\left(j_{1}, \ldots, j_{n}\right)}\left(\sum_{k=1}^{n} H_{n, k}^{\left(j_{k}\right)} \cdot \frac{k}{k}\right)^{2} \leq \max _{\left(j_{1}, \ldots, j_{n}\right)} \sum_{k=1}^{n}\left(H_{n, k}^{\left(j_{k}\right)}\right)^{2} k^{2} \sum_{k=1}^{n} k^{-2} \\
& \leq 2 \sum_{k=1}^{n} k^{2} \sum_{j=1}^{2^{k}}\left(H_{n, k}^{(j)}\right)^{2} .
\end{aligned}
$$

Put, for $\nu=1,2,3,4$,

$$
A_{\nu}^{(N)}=\left\{\sum_{n=1}^{N} \sum_{k=1}^{n} k^{2} \sum_{j=1}^{2^{k}}\left(\int_{\Gamma_{\nu}} R_{n, k, j}(\lambda) d E_{\lambda} \xi\right)^{2}\right\}^{1 / 2}
$$


where

$$
\begin{array}{ll}
\Gamma_{1}=\left[1-2^{-n}, 1\right), & \Gamma_{2}=\left[1-2^{-n+k}, 1-2^{-n}\right), \\
\Gamma_{3}=\left[0,1-2^{-n-k}\right), & \Gamma_{4}=(-\infty, 0) .
\end{array}
$$

Clearly, to prove (25) it is enough to show that

$$
\left\|A_{\nu}^{(N)}\right\|_{r} \leq C_{r}\|\xi\|_{r}+D<\infty, \quad N=1,2, \ldots, \text { for } \nu=1,2,3,4 .
$$

In the general case, the argument will be similar to the above. Namely, we first approximate the integrals $\int_{\Gamma_{\nu}} R d E_{\lambda} \xi$ by Riemann-Stieltjes sums. Then we apply inequalities for suitable martingales and their square functions. Finally, using the estimates for $B(n, x)$ and interpolating some operators in $\mathbb{L}^{p}$-spaces we get $(30)$ for $\nu=1, \ldots, 4$.

We proceed to the proof of $(30)$.

For a partition $\pi=\left(0=t_{0}<\cdots<t_{n}=1\right)$ of $[0,1]$ and a function defined on $[0,1]$, we set, as before,

$$
\pi(f)=\sum f\left(t_{s}\right)\left(E_{t_{s}}-E_{t_{s-1}}\right) \xi \quad \text { and } \quad I(f)=\int_{0}^{1} f(\lambda) d E_{\lambda} \xi
$$

Fix a sequence $\pi_{N}=\left(0=t_{1}^{(N)}<\cdots<t_{k(N)}^{(N)}=1\right)$ of partitions such that

$$
\sum_{\nu=1}^{3}\left\|\left\{\sum_{n=1}^{N} \sum_{k=1}^{n} k^{2} \sum_{j=1}^{2^{k}}\left[\pi_{N}\left(R_{n, k, j} \chi_{\Gamma_{\nu}}\right)-I\left(R_{n, k, j} \chi_{\Gamma_{\nu}}\right)\right]^{2}\right\}^{1 / 2}\right\|_{r} \leq D<\infty
$$

for $N=1,2, \ldots$ Then for $A_{\nu}^{(N)}$ defined in (28) we have the estimate

$$
\begin{aligned}
& \left\|A_{\nu}^{(N)}\right\|_{r} \\
\leq & \left\|\left\{\sum_{n=1}^{N} \sum_{k=1}^{n} k^{2} \sum_{j=1}^{2^{k}}\left(\sum_{t_{s}^{(N)} \in \Gamma_{\nu}} R_{n, k, j}\left(t_{s}^{(N)}\right)\left(E_{t_{s}^{(N)}}-E_{t_{s-1}^{(N)}}\right) \xi\right)^{2}\right\}^{1 / 2}\right\|_{r}+D
\end{aligned}
$$

for $\nu=1,2,3$ and $N=1,2, \ldots$ We write briefly $\Delta_{s}=\left(E_{t_{s}^{(N)}}-E_{t_{s-1}^{(N)}}\right) \xi$. Consequently, to prove $(30)$, for $\nu=1,2,3$, it is enough to show that

$$
G_{\nu}=\left\|\left\{\sum_{n=1}^{N} \sum_{k=1}^{n} k^{2} \sum_{j=1}^{2^{k}}\left(\sum_{t_{s}^{(N)} \in \Gamma_{\nu}} R_{n, k, j}\left(t_{s}^{(N)}\right) \Delta_{s}\right)^{2}\right\}^{1 / 2}\right\|_{r} \leq C_{r}\|\xi\|_{r}
$$

for $N=1,2, \ldots$ and $\nu=1,2,3$.

We proceed to the proof of (32).

CASE $\nu=1$. Writing

$$
\left[1-2^{-n}, 1\right)=\bigcup_{\alpha=0}^{\infty}\left[1-2^{-n-\alpha}, 1-2^{-n-\alpha-1}\right)=\bigcup_{\alpha=0}^{\infty} I_{n, \alpha},
$$


by the triangle inequality we get

$$
G_{1} \leq \sum_{\alpha=0}^{\infty} \sum_{k=1}^{N} k\left\|\left\{\sum_{n=k}^{N} \sum_{j=1}^{2^{k}}\left(\sum_{t_{s}^{(N)} \in I_{n, \alpha}} R_{n, k, j}\left(t_{s}^{(N)}\right) \Delta_{s}\right)^{2}\right\}^{1 / 2}\right\|_{r}
$$

Fixing $\alpha, k, j$, we consider the martingale

$$
Y_{\varrho}^{(\alpha, k, j)}=\sum_{s=1}^{\varrho} \psi_{\alpha, k, j}(s) \Delta_{s},
$$

where $\psi_{\alpha, k, j}(s)=\sum_{n=k}^{N} \sum_{t_{s}^{N} \in I_{n, \alpha}} R_{n, k, j}\left(t_{s}^{(N)}\right)$. Using the estimate

$$
\left|C_{n}(\lambda)-C_{m}(\lambda)\right| \leq C(1-\lambda)|n-m|,
$$

we get

$$
\sup _{\varrho}\left\|Y_{\varrho}^{\alpha, k, j}\right\|_{r} \leq C 2^{-k-\alpha}\|\xi\|_{r}, \quad 1<r<\infty .
$$

Let $\left\{m_{n}^{(\alpha)}\right\}$ be a sequence of positive integers such that $t_{s}^{(N)} \in I_{n, \alpha}$ is equivalent to $m_{n}^{(\alpha)}<s \leq m_{n+1}^{(\alpha)}$. Putting $B_{n}^{(\alpha, k, j)}=Y_{m_{n}^{(\alpha)}}^{(\alpha, k, j)}$, and taking for the martingale $\left(B_{n}^{(\alpha, k, j)}\right)_{n=k}^{N}$ its square function

$$
Q^{(\alpha, k, j)}=\left\{\sum_{n=k}^{N}\left(\sum_{t_{s}^{N} \in I_{n, \alpha}} R_{n, k, j}\left(t_{s}^{(N)}\right) \Delta_{s}\right)^{2}\right\}^{1 / 2},
$$

we infer, by the Burkholder inequality and (34), that

$$
\left\|Q^{(\alpha, k, j)}\right\|_{r} \leq C_{r} 2^{-\alpha-k}\|\xi\|_{r} .
$$

For $r=2$,

$$
\left\|\left\{\sum_{n=k}^{N} \sum_{j=1}^{2^{k}}\left(\sum_{t_{s}^{N} \in I_{n, \alpha}} R_{n, k, j}\left(t_{s}^{(N)}\right) \Delta_{s}\right)^{2}\right\}^{1 / 2}\right\|_{2} \leq C 2^{-k / 2} 2^{-\alpha} .
$$

To interpolate between (35) and (36) we define (for $p$ such that $r$ lies between 2 and $p$ ) the operator

$$
W_{\alpha, k}^{(p)}: \mathbb{L}^{p}(\Omega, \mu) \rightarrow \mathbb{L}^{p}\left((\Omega, \mu), \ell_{\left(\begin{array}{l}
k \leq n \leq N \\
1 \leq j \leq 2^{k}
\end{array}\right)}^{2}\right),
$$

by putting

$$
\left.W_{\alpha, k}^{(p)}: \xi \mapsto\left\{\sum_{t_{s}^{N} \in I_{n, \alpha}} R_{n, k, j}\left(t_{s}^{(N)}\right) \Delta_{s} \xi\right\}\right\}_{\substack{k \leq n \leq N \\ 1 \leq j \leq 2^{k}}}
$$

Then

$$
\left\|W_{\alpha, k}^{(p)}\right\|_{r} \leq C 2^{-\alpha}, \quad\left\|W_{\alpha, k}^{(2)}\right\|_{2} \leq C 2^{-\alpha-k / 2} .
$$


Interpolation gives

$$
\left\|W_{\alpha, k}^{(r)}\right\|_{r} \leq C_{r} 2^{-\alpha} 2^{-\alpha-k / 2 \theta_{p}}, \quad 0<\theta_{p} \leq 1 .
$$

Summing up over $k$ and $\alpha$ we finally get (32) for $\nu=1$.

The argument for $\nu=2$ and $\nu=3$ is similar, so we only sketch it.

CASE $\nu=2$. We write

$$
\left[1-2^{-n+k}, 1-2^{-n}\right)=\bigcup_{\alpha=1}^{k}\left[1-2^{-n+\alpha}, 1-2^{-n+\alpha-1}\right)=\bigcup_{\alpha=1}^{k} J_{n, \alpha} .
$$

Then

$$
G_{2} \leq \sum_{k=1}^{N} \sum_{\alpha=1}^{k} k\left\|\left\{\sum_{j=1}^{2^{k}} \sum_{n=k}^{N}\left(\sum_{t_{s}^{N} \in I_{n, \alpha}} R_{n, k, j}\left(t_{s}^{(N)}\right) \Delta_{s}\right)^{2}\right\}^{1 / 2}\right\|_{r} .
$$

We set

$$
\psi_{\alpha, k, j}(s)=\sum_{n=k}^{N} \sum_{t_{s}^{N} \in J_{n, \alpha}} R_{n, k, j}\left(t_{s}^{(N)}\right)
$$

and define the martingale $Y_{\varrho}^{(\alpha, k, j)}$ as in (33).

Using the estimate

$$
\left|C_{n}-C_{m}\right| \leq C m^{-1}(n-m) \quad \text { for } n>m,
$$

martingale inequalities and interpolation we can imitate the previous argument used for $G_{1}$, to obtain (32) for $\nu=2$.

CASE $\nu=3$. We write

$$
\left[0,1-2^{-n+k}\right)=\bigcup_{\alpha=0}^{n-k-1}\left[1-2^{-n+k+\alpha+1}, 1-2^{-n+k+\alpha}\right)=\bigcup_{\alpha=0}^{n-k-1} I_{n, \alpha, k} .
$$

Then

$$
G_{3} \leq\left\|\left\{\sum_{k=1}^{N} \sum_{n=k}^{N} k^{2} \sum_{j=1}^{2^{k}}\left(\sum_{\alpha=0}^{n-k-1} \sum_{t_{s}^{N} \in I_{n, \alpha, k}} R_{n, k, j}\left(t_{s}^{(N)}\right) \Delta_{s}\right)^{2}\right\}^{1 / 2}\right\|_{r} .
$$

It is convenient to take $0 \leq \alpha<\infty$ and write the estimate

$$
G_{3} \leq \sum_{\alpha=0}^{\infty} \sum_{k=1}^{N} k\left\|\left\{\sum_{n=k}^{N} \sum_{j=1}^{2^{k}}\left(\sum_{t_{s}^{(N)} \in I_{n, \alpha, k}} R_{n, k, j}\left(t_{s}^{(N)}\right) \Delta_{s}\right)^{2}\right\}^{1 / 2}\right\|_{r} .
$$

Putting

$$
\psi_{\alpha, k, j}(s)=\sum_{n=k}^{N} \sum_{t_{s}^{(N)} \in I_{n, \alpha, k}} R_{n, k, j}\left(t_{s}^{(N)}\right)
$$


and taking a suitable martingale transform we can follow the previous argument, using the estimate $\left|C_{n}\right| \leq C n^{-1}(1-\lambda)^{-1}$.

In this way we get $(32)$ for $\nu=3$.

It remains to prove $(30)$ in the case $\nu=4$. The argument here is much easier, because, for $\lambda>0,|B(m, \lambda)| \leq e^{-m}$. This implies the estimate

$$
\begin{aligned}
& \|\left\{\sum_{n=1}^{N} \sum_{k=1}^{n} k^{2} \sum_{j=1}^{2^{k}}\right.\left.\left(\int_{-\infty}^{0} R_{n, k, j}(\lambda) d E_{\lambda} \xi\right)^{2}\right\}^{1 / 2} \|_{r} \\
& \leq C \sum_{n=1}^{\infty} \sum_{k=1}^{n} k^{2} 2^{k} e^{-2^{n}}\|\xi\|_{r} \leq C\|\xi\|_{r}, \quad N=1,2, \ldots
\end{aligned}
$$

The proof of the theorem is complete.

\section{References}

[1] J. Bergh and J. Löfström, Interpolation Spaces, Springer, 1976.

[2] E. Berkson, J. Bourgain and T. A. Gillespie, On the almost everywhere convergence of ergodic averages for power bounded operators on $L^{p}$-subspaces, Integral Equations Operator Theory 14 (1991), 678-715.

[3] E. Berkson and T. A. Gillespie, Stečkin's theorem, transference and spectral decompositions, J. Funct. Anal. 70 (1987), 140-170.

[4] O. Bratteli and D. W. Robinson, Operator Algebras and Quantum Statistical Mechanics, Vol. 1, Springer, New York, 1979.

[5] D. L. Burkholder, Martingale transforms, Ann. Math. Statist. 37 (1966), 1494-1505.

[6] R. H. Dowson, Spectral Theory of Linear Operators, London Math. Soc. Monogr. 12, Academic Press, New York, 1978.

[7] R. E. Edwards and G. I. Gaudry, Littlewood-Paley and Multiplier Theory, Ergeb. Math. Grenzgeb. 90, Springer, Berlin, 1977.

[8] V. F. Gaposhkin, Criteria for the strong law of large numbers for some classes of weakly stationary processes and homogeneous random fields, Theory Probab. Appl. 22 (1977), 286-310.

[9] - Individual ergodic theorem for normal operators in $L^{2}$, Funktsional. Anal. i Prilozhen. 15 (1981), no. 1, 18-22 (in Russian).

[10] A. Garsia, Topics in Almost Everywhere Convergence, Markham, Chicago, 1970.

[11] R. Jajte, Pointwise ergodic theorem for unbounded operators in $\mathbb{L}^{2}$, Teor. Veroyatnost. Primenen. 50 (2005), 1-19.

[12] - Operators on $\mathbb{L}^{p}$-spaces determined by filtrations and pointwise ergodic theorems, submitted.

[13] R. Jajte and A. Paszkiewicz, Almost sure approximation of unbounded operators in $\mathbb{L}^{2}(X, \mathcal{A}, \mu)$, Studia Math. 128 (1998), 103-120.

[14] - - - Topics in almost sure approximation of operators in $\mathbb{L}^{2}$-spaces, in: Interaction between Functional Analysis, Harmonic Analysis, and Probability, N. Kalton et al. (eds.), Dekker, New York, 1996, 219-228. 
[15] P. W. Millar, Martingale integrals, Trans. Amer. Math. Soc. 133 (1968), 145-166.

[16] E. Nelson, Analytic vectors, Ann. of Math. 70 (1959), 572-614.

Faculty of Mathematics

University of Łódź

Banacha 22

90-238 Łódź, Poland

E-mail: rjajte@math.uni.lodz.pl

Received January 27, 2006

Revised version December 28, 2006

$(5851)$ 